\title{
Meroterpenoid-Rich Fraction of the Ethanolic Extract from Sargassum serratifolium Suppressed Oxidative Stress Induced by Tert-Butyl Hydroperoxide in HepG2 Cells
}

\author{
Sujin Lim ${ }^{1}$, Misung Kwon ${ }^{1}$, Eun-Ji Joung ${ }^{1}$, Taisun Shin ${ }^{2}$, Chul-Woong Oh ${ }^{3}$, Jae Sue Choi ${ }^{1} \mathbb{C}$ \\ and Hyeung-Rak Kim ${ }^{1, *}$ \\ 1 Department of Food Science and Nutrition, Pukyong National University, 45, Yongso-Ro, Nam-Gu, \\ Busan 48513, Korea; cocky0305@naver.com (S.L.); mskwon80@hanmail.net (M.K.); \\ eunji2007@naver.com (E.-J.J.); choijs@pknu.ac.kr (J.S.C.) \\ 2 Division of Food and Nutrition, Chonnam National University, 77, Yongbong-ro, Buk-gu, \\ Gwangju 61186, Korea; shints@jnu.ac.kr \\ 3 Department of Marine Biology, Pukyong National University, 45, Yongso-Ro, Nam-Gu, Busan 48513, Korea; \\ ohcw@pknu.ac.kr \\ * Correspondence: hrkim@pknu.ac.kr; Tel.: +82-51-629-5847; Fax: +82-51-629-5842
}

Received: 6 August 2018; Accepted: 7 October 2018; Published: 9 October 2018

\begin{abstract}
Sargassum species have been reported to be a source of phytochemicals, with a wide range of biological activities. In this study, we evaluated the hepatoprotective effect of a meroterpenoid-rich fraction of the ethanolic extract from Sargassum serratifolium (MES) against tert-butyl hydroperoxide ( $t$-BHP)-treated HepG2 cells. Treatment with MES recovered the cell viability from the $t$-BHP-induced oxidative damage in a dose-dependent manner. It suppressed the reactive oxygen species production, lipid peroxidation, and glutathione depletion in the $t$-BHP-treated HepG2 cells. The activity of the antioxidants induced by $t$-BHP, including superoxide dismutase (SOD) and catalase, was reduced by the MES treatment. Moreover, it increased the nuclear translocation of nuclear factor erythroid 2-related factor 2, leading to the enhanced activity of glutathione $S$ transferase, and the increased production of heme oxygenase- 1 and $\mathrm{NAD}(\mathrm{P}) \mathrm{H}$ :quinine oxidoreductase 1 in $t$-BHP-treated HepG2 cells. These results demonstrate that the antioxidant activity of MES substituted the activity of the SOD and catalase, and induced the production of detoxifying enzymes, indicating that MES might be used as a hepatoprotectant against $t$-BHP-induced oxidative stress.
\end{abstract}

Keywords: antioxidant; oxidative stress; Sargassum serratifolium; tert-butyl hydroperoxide; Nrf2

\section{Introduction}

Reactive oxygen species (ROS) are continuously generated in organisms as a by-product of aerobic respiration. They play a key physiological role in the stimulation of growth and immune response in living cells in response to intracellular and extracellular stimuli [1]. The ROS level is regulated by enzymatic and non-enzymatic antioxidant defense systems. Excessive ROS are instantly removed by intracellular antioxidant compounds, such as glutathione (GSH), vitamin C, and vitamin E, and are perpetually regulated by antioxidant enzymes, including superoxide dismutase (SOD), catalase, glutathione reductase, glutathione peroxidase, and glutathione $S$-transferase (GST) [2]. Reactive oxygen species damage major cellular biomolecules, such as DNA, protein, and lipids, leading to pathophysiological conditions, including diabetes mellitus, hypertension, obesity, dyslipidemia, cancer, and inflammation [3]. Thus, dietary supplementation of antioxidants has been used as a preventive or therapeutic strategy against oxidative damage caused by ROS. 
The nuclear factor (erythroid-derived 2)-like 2 (Nrf2) is a basic region/leucine zipper transcription factor, which regulates the expression of antioxidants and detoxifying enzymes [heme oxygenase-1 (HO-1), NAD(P)H:quinone acceptor oxidoreductase 1 (NQO1), and GST]. Under basal conditions, Nrf2 is inactive in the cytosol by binding with Kelch-like ECH-associated protein 1 (Keap1), which inhibits the translocation of Nrf2 from the cytosol into the nucleus [4]. The dissociation of Nrf2 from its cytosolic repressor, Keap1, is a primary step in the activation of Nrf2, which induces the expression of phase II detoxifying enzymes [5]. NQO1, HO-1, and GST have been known to be target enzymes of Nrf2. NQO1 reduces quinone to hydroquinone by a detoxification reaction in biological systems [6]. HO-1 induces the enzymatic degradation of heme to produce carbon monoxide and biliverdin, which is subsequently converted into bilirubin, a strong antioxidant [7].

Tert-butyl hydroperoxide ( $t$-BHP) is a well-known pro-oxidant, which induces oxidative damage in in vivo or in vitro models. The metabolic intermediates of $t$-BHP by cytochrome P-450 in hepatocytes generate toxic free radicals, such as peroxyl and alkoxyl radicals, resulting in oxidative damage to the cells [8]. Thus, $t$-BHP is widely used to induce oxidative damage in order to investigate the mechanism of hepatocyte injury [9]. HepG2 cells, a human hepatoma cell line, have been used to study the mechanisms of oxidative stress and xenobiotics metabolism, as they retain their endogenous antioxidants and xenobiotic-metabolizing enzymes [10].

Brown algae are a rich source of bioactive compounds, such as meroterpenoids, phlorotannins, fucoxanthin, sterols, and glycolipids [11,12]. Sargassum serratifolium (C. Agardh) is distributed throughout the coast of Korea and Japan. Recently, we found that the ethanolic extract of S. serratifolium exhibits a strong anti-inflammatory activity, and the active compounds involved were identified as sargahydroquinoic acid (SHQA), sargachromanol (SCM), and sargaquinoic acid (SQA) [13,14]. We obtained a meroterpenoid-rich fraction from the ethanolic extract of $S$. serratifolium (MES) by removing the salts and water-soluble saccharides. The combined amount of SHQA, SCM, and SQA in $100 \mathrm{~g}$ of MES was estimated to be $45.7 \pm 2.2 \mathrm{~g}$, indicating that MES contains a high amount of active components $[15,16]$. In this study, we investigated the hepatoprotective effect of MES on $t$-BHP-treated HepG2 cells, and confirmed that MES induced antioxidants and detoxifying enzymes by activating Nrf2. The findings in this study support that MES may be used as a source of nutraceuticals for hepatoprotection.

\section{Results}

\subsection{Hepatoprotective Effect of MES}

To determine the non-toxic concentration range of MES in HepG2 cells, the MTS assay was performed with various concentrations of MES. As shown in Figure 1A, MES had no cytotoxicity at a concentration up to $10.0 \mu \mathrm{g} / \mathrm{mL}$. The cells were treated with different concentrations of MES $(0-1.0 \mu \mathrm{g} / \mathrm{mL})$ in the presence of $0.5 \mathrm{mM} t$-BHP in order to evaluate the hepatoprotective effect of MES on $t$-BHP-treated cells. Treatment with $t$-BHP $(0.5 \mathrm{mM})$ decreased the viability of HepG2 cells to $65 \%$. However, treatment with MES recovered the cell viability in a dose-dependent manner (Figure 1B). Furthermore, treatment with $1.0 \mathrm{\mu g} / \mathrm{mL}$ MES completely recovered the viability of the cells damaged by $t$-BHP. N-acetyl-L-cysteine (NAC) $(0.1 \mathrm{mM})$, as a positive control, showed an $80 \%$ of cell viability. 


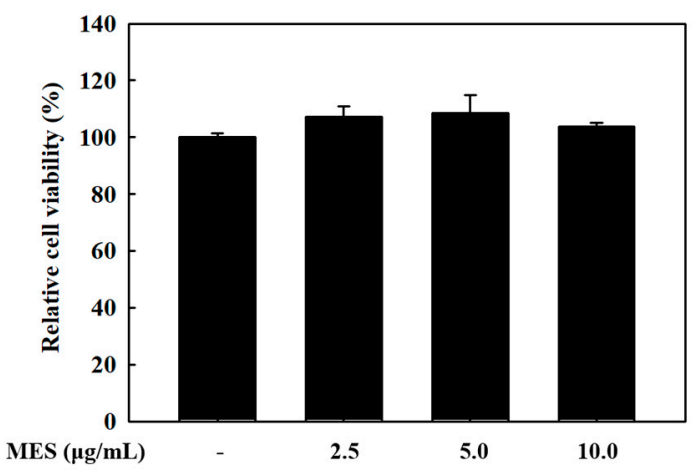

(A)

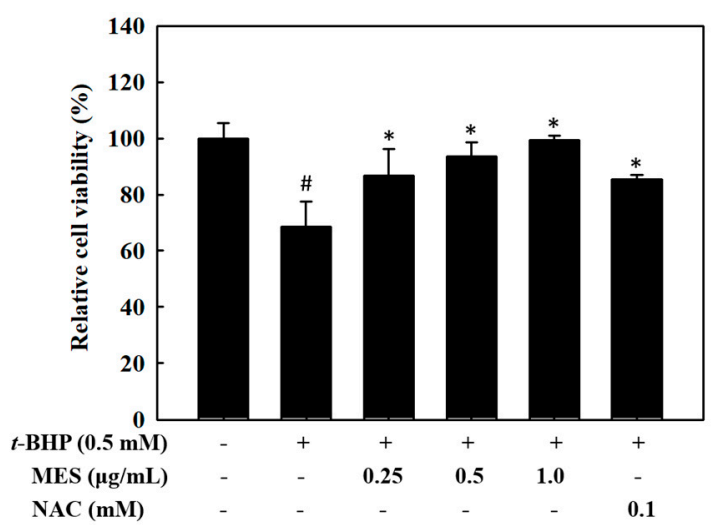

(B)

Figure 1. Hepatoprotective effect of MES on $t$-BHP-stimulated oxidative stress. (A) The cell viability of MES was measured by using Cell Proliferation Assay Kit. (B) The hepatoprotective effect was determined with various concentrations of MES in $t$-BHP-treated HepG2 cells. The data are the means $\pm \mathrm{SD}$ of three independent experiments. $\# p<0.05$ indicates significant differences from the control group. ${ }^{*} p<0.05$ indicates significant differences from the $t$-BHP treatment group.

\subsection{Effect of MES on the Inhibition of ROS Production and Lipid Peroxidation}

We measured the intracellular ROS levels by using DCFH-DA to evaluate whether MES inhibits ROS production in $t$-BHP-treated HepG2 cells. As shown in Figure 2A, MES inhibited the $t$-BHP-induced ROS production in HepG2 cells in a dose-dependent manner. The ROS level in the $1.0 \mu \mathrm{g} / \mathrm{mL}$ MES treatment group was similar to that in the control group, which was significantly lower than that in the $0.1 \mathrm{mM}$ NAC group. However, the reduction of the ROS level by MES-only treatment was negligible. To determine whether MES suppresses lipid peroxidation, we measured the intracellular MDA level with various concentrations of MES in the presence of $0.5 \mathrm{mM} t$-BHP. The increased MDA level by $t$-BHP treatment was reduced by MES treatment in a dose-dependent manner (Figure 2B).

\subsection{Effect of MES on the Glutathione Levels}

We measured the change of GSH levels using the fluorescent probe CMF-DA, as glutathione is a primary intracellular antioxidant molecule against oxidative stress. The decreased GSH level by $t$-BHP treatment was recovered by MES treatment in a dose-dependent manner (Figure 2C), indicating that MES may prevent the oxidation of GSH.

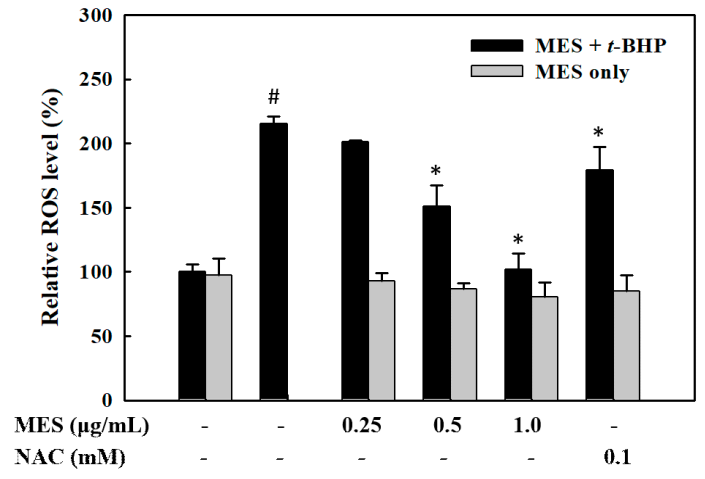

(A)

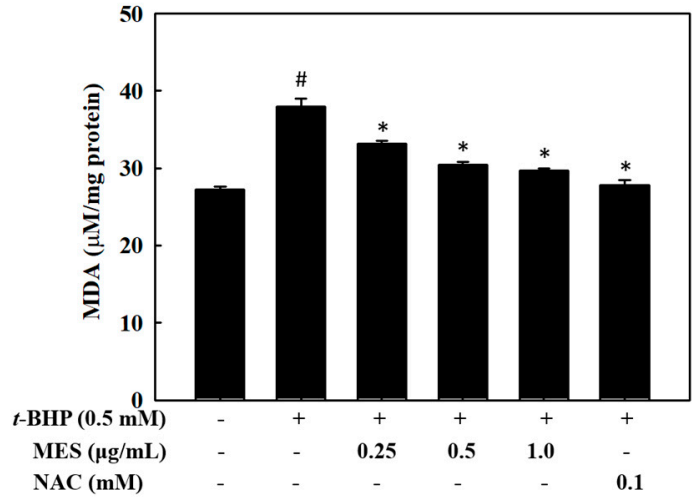

(B)

Figure 2. Cont. 


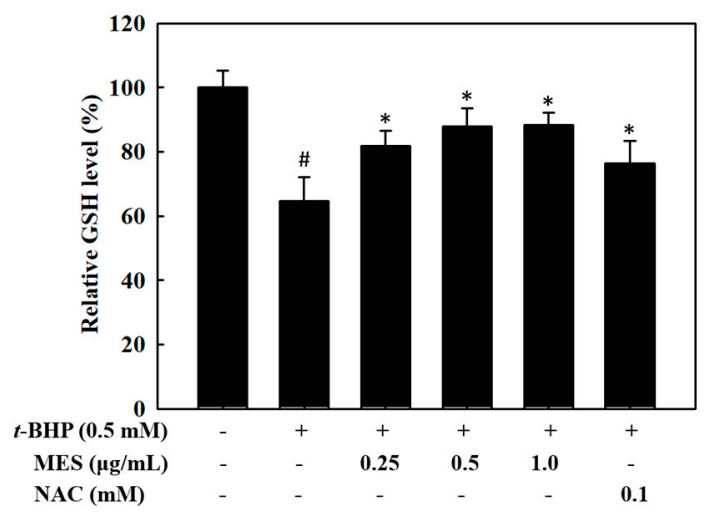

(C)

Figure 2. Inhibitory effect of MES on the reactive oxygen species generation, lipid peroxidation, and GSH depletion in $t$-BHP-treated HepG2 cells. HepG2 cells were treated with MES $(0.25 \sim 1.0 \mu \mathrm{g} / \mathrm{mL})$ and $t$-BHP. (A) ROS were measured by a fluorescent probe, DCFH-DA. (B) Lipid peroxidation was measured by using a TBARS assay. (C) The GSH level was measured by fluorescent probe, CMF-DA. The data are means \pm SD of three independent experiments. $\# p<0.05$ indicates significant differences from the control group. ${ }^{*} p<0.05$ indicates significant differences from the $t$-BHP treatment group.

\subsection{Effect of MES on SOD and Catalase Activities}

The antioxidant enzymes, such as SOD and catalase, play a crucial role in the removal of the free radicals induced by oxidative damage. To determine the influence of MES on the antioxidant enzyme activities, we determined the activities of SOD (Figure 3A) and catalase (Figure 3B) after $24 \mathrm{~h}$ of treatment. As shown in Figure 3, the $t$-BHP treatment induced significantly increased activities of SOD and catalase $(p<0.05)$. However, the MES treatment reduced both the SOD and catalase activities in a dose-dependent manner, indicating an intrinsic antioxidant activity of the MES compensates both enzyme activities. The $N$-acetyl-L-cysteine $(0.1 \mathrm{mM})$ did not recover the activity of SOD, whereas, it recovered the activity of the catalase.

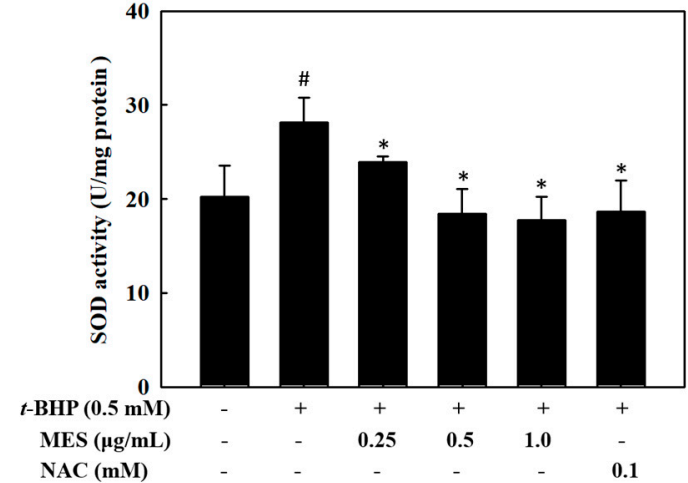

(A)

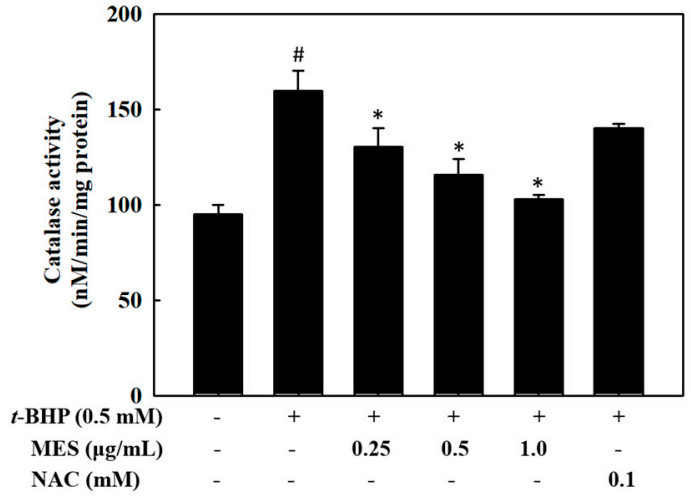

(B)

Figure 3. Effect of MES on antioxidant enzyme activities in t-BHP-treated HepG2 cells. Cells were treated with MES and $t$-BHP for $18 \mathrm{~h}$. The cell lysates were prepared and used for superoxide dismutase (A) and catalase (B) activities. The values are the means \pm SD of three independent experiments. $\# p<0.05$ indicates significant differences from the control group. ${ }^{*} p<0.05$ indicates significant differences from the $t$-BHP treatment group.

\subsection{Effect of MES on GST Activity and Expression of HO-1 and NQO1}

The activity of the detoxifying enzymes, such as GST, HO-1, and NQO1, was measured to confirm whether the detoxifying enzymes are involved in the increased viability of the $t$-BHP-treated HepG2 cells. The activity of the GST, reduced by $t$-BHP treatment, was recovered by treatment with MES in 
a dose-dependent manner (Figure 4A), however the expression of HO-1 and NQO1 was not affected by the $t$-BHP treatment. Particularly, the expression of HO- 1 was increased by the highest concentration of the MES, while that of NQO1 was induced at a low concentration of MES (Figure 4B). The expression level of HO-1 by $1.0 \mathrm{\mu g} / \mathrm{mL}$ MES was enhanced four-fold compared with that in the control group. Moreover, $0.1 \mathrm{mM}$ NAC (positive control) enhanced the expression of HO-1, but not the expression of NQO1.

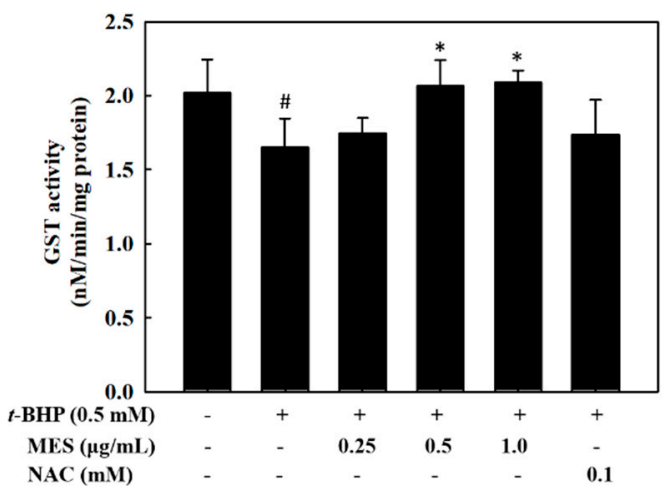

(A)

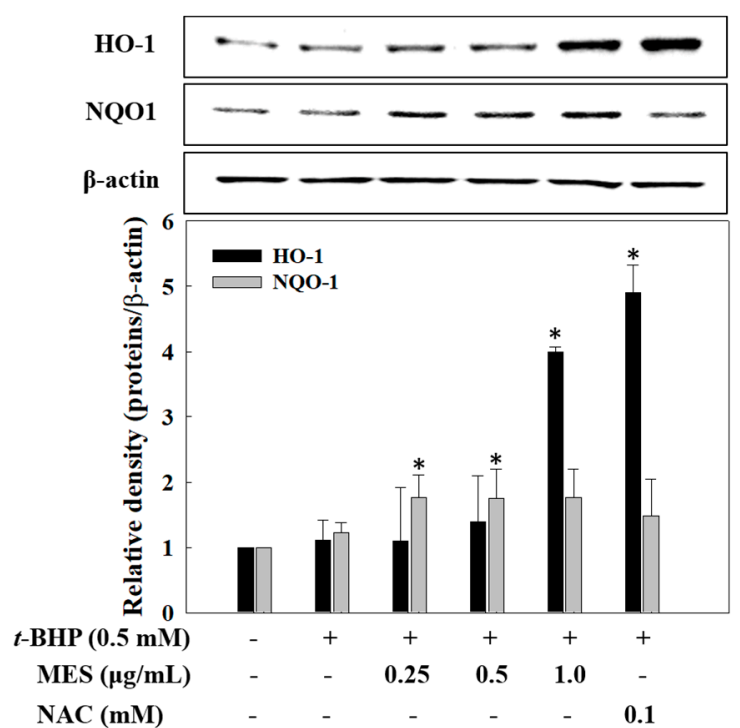

(B)

Figure 4. Effect of MES on the activity of GST and the productions of HO-1 and NQO1 in $t$-BHP-treated HepG2 cells. Cells were treated with MES and $t$-BHP for $18 \mathrm{~h}$. (A) The GST activity was measured with a glutathione S-transferase assay kit. (B) The expressions of HO-1 and NQO1 were analyzed by Western blot. The data are means \pm SD of three independent experiments. \# $p<0.05$ indicates significant differences from the control group. ${ }^{*} p<0.05$ indicates significant differences from the $t$-BHP treatment group.

\subsection{Effect of MES on Nrf2 Expression and Nuclear Translocation}

As GST, HO-1, and NQO1 are regulated by the transcription factor Nrf2, we determined the translocation of Nrf2 into the nucleus after the treatment with MES. Cell lysates were separated into cytosolic and nuclear fractions, and the level of Nrf2 in each fraction was determined by Western blot. As shown in Figure 5A, the Nrf2 levels in the nuclear fraction were increased by MES treatment. To examine the effect of MES on the tranlocation of Nrf2 in the presence of $t$-BHP, the separated cytosolic and nucleic fractions were used to analyze the level of Nrf2. As shown in Figure 5B, the level of Nrf2 in the cytosol was significantly reduced in a dose-dependent manner $(p<0.05)$, whereas, the level of Nrf2 in the nuclear fraction increased. The increased level of Nrf2 by MES in the presence of $t$-BHP was higher than that by MES only. The level of Keap1 in the cytosol also decreased dose-dependently, inducing the Nrf2 translocation. Like MES, $0.1 \mathrm{mM}$ NAC (positive control) enhanced the translocation of Nrf2 into the nucleus. We further confirmed the translocation of Nrf2 from the cytosol into the nucleus by confocal microscopy. In the control and $t$-BHP-treated cells, Nrf2 (red) was mostly observed in the cytosol. After stimulation by MES, Nrf2 was densely detected in the nucleus (Figure 5B). This result indicates that MES activates the transcriptional activity of Nrf2 via translocation into the nucleus in HepG2, suggesting that MES induces the expression of phase II detoxifying enzymes through the activation of Nrf2. 


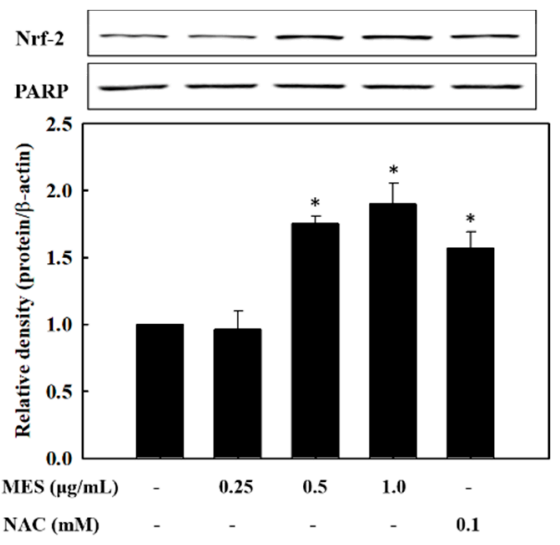

(A)

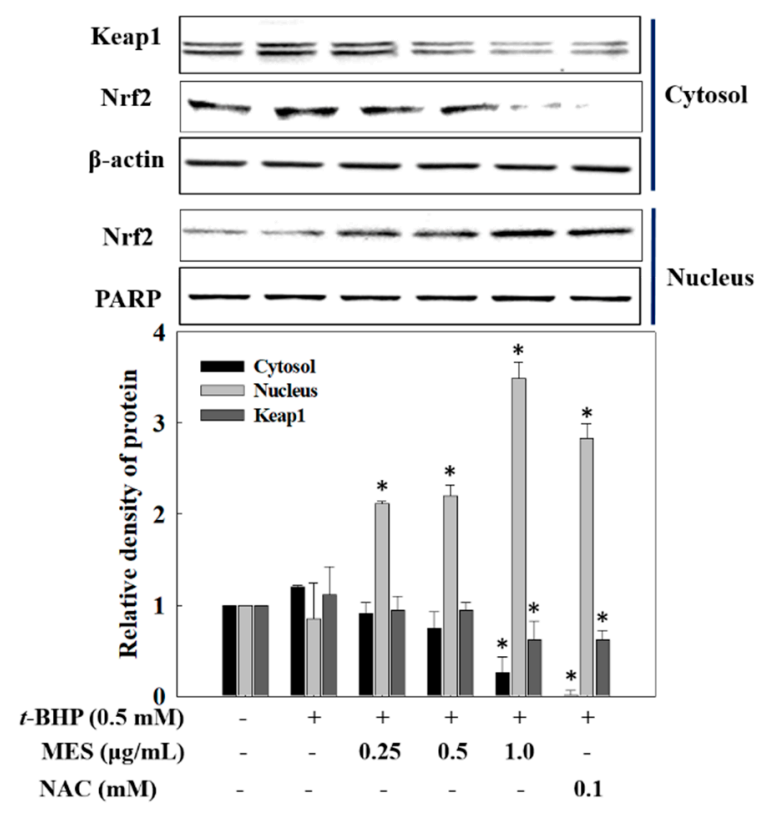

(B)

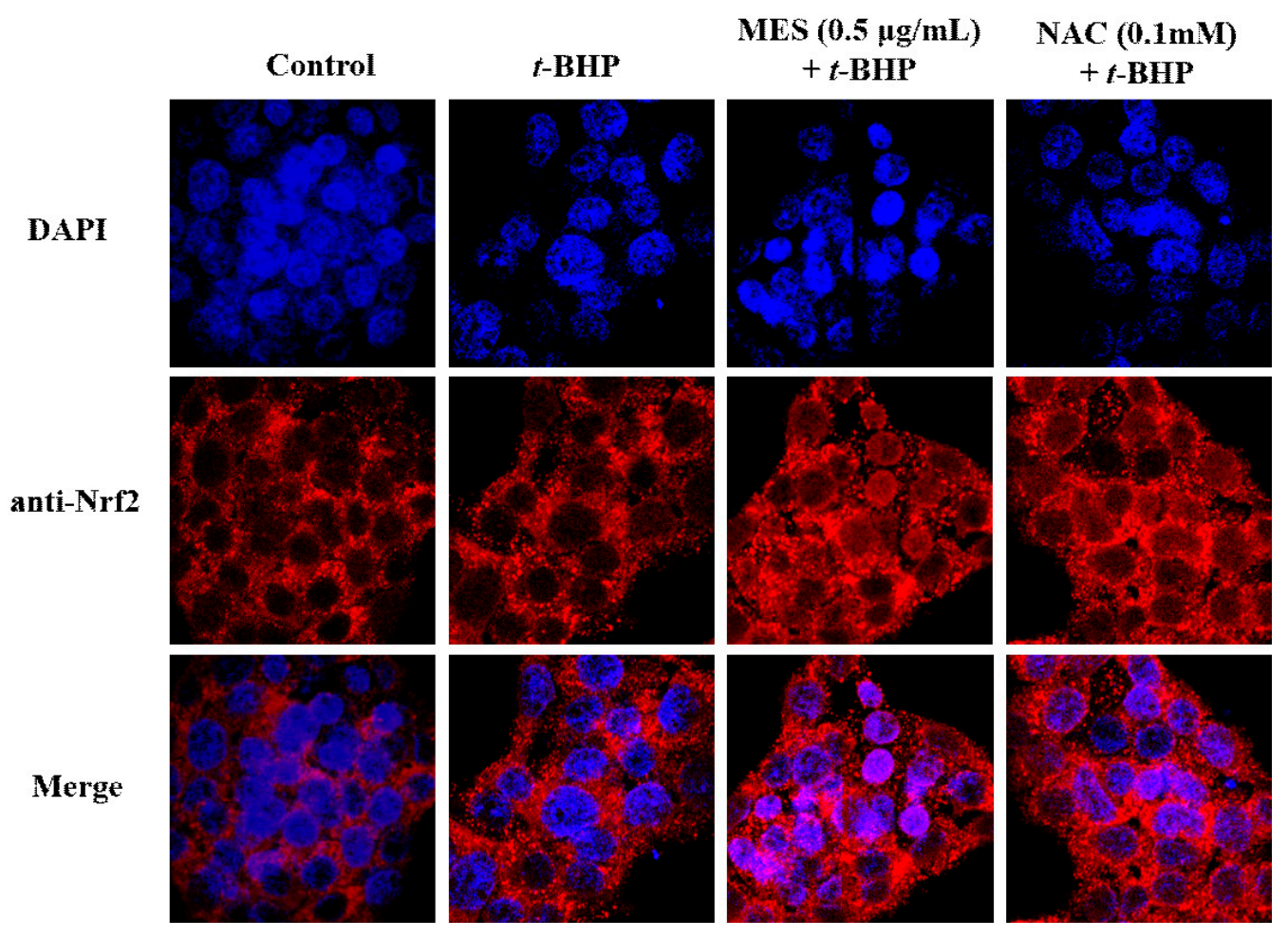

(C)

Figure 5. Effect of MES on the activation and translocation of Nrf2. (A) Cells were treated with indicated concentrations of MES for $1 \mathrm{~h}$, and the nuclear fraction was analyzed with Western blot. (B) The cells were treated with MES and $t$-BHP for $1 \mathrm{~h}$ and separated cytosolic and nuclear fraction were analyzed with Western blot. (C) Cells were fixed and immunostained with anti-Nrf2 antibody (Alexa Fluor 586) and DAPI. The images were captured by confocal microscopy. The data are means $\pm \mathrm{SD}$ of three independent experiments. ${ }^{*} p<0.05$ indicates significant differences from the $t$-BHP treatment group. 


\subsection{Identification of Antioxidant Components in MES}

To identify the antioxidant components in MES, we isolated SHQA, SCM, and SQA as described before [14]. The contents of SHQA, SCM, and SQA in $100 \mathrm{~g}$ of MES were estimated to be $37.6 \pm 2.1$, $6.23 \pm 0.36$, and $1.89 \pm 0.10 \mathrm{~g}$, respectively (Table 1 ). The inhibitory activity $\left(\mathrm{IC}_{50}\right)$ of these components on the ROS production was determined using t-BHP-stimulated HepG2 cells. As shown in Table 1, the $\mathrm{IC}_{50}$ values of MES, SHQA, SCM, and SQA for the inhibition of ROS were $0.52 \pm 0.08,0.38 \pm 0.04$, $0.52 \pm 0.12$, and $0.35 \pm 0.03 \mu \mathrm{g} / \mathrm{mL}$, respectively.

Table 1. Composition and inhibitory activities of MES and isolated components on reactive oxygen species (ROS) in tert-butyl hydroperoxide (t-BHP)-stimulated HepG2 cells.

\begin{tabular}{ccc}
\hline Compounds & IC $_{\mathbf{5 0}}{ }^{\mathbf{a}}(\boldsymbol{\mu g} / \mathbf{m L})$ & Composition $(\%)$ \\
\hline MES & $0.52 \pm 0.08$ & 100 \\
Sargahydroquinoic acid & $0.38 \pm 0.04$ & $37.6 \pm 2.10$ \\
Sargachromenol & $0.52 \pm 0.12$ & $6.23 \pm 0.36$ \\
Sargaquinoic acid & $0.35 \pm 0.03$ & $1.89 \pm 0.10$ \\
\hline
\end{tabular}

${ }^{\mathrm{a}} \mathrm{IC}_{50}$ was measured with inhibition of ROS production by DCFH-DA fluorescence probe in $t$-BHP-induced HepG2 cells. ${ }^{\mathrm{b}}$ Composition shows amounts of active compounds in $100 \mathrm{~g}$ of MES.

\section{Discussion}

Oxidative damage is caused by an imbalance between the ROS level and antioxidant capacity in organisms. Recent studies have suggested that excess free radicals play a key role in the development and progression of liver injuries [17]. Thus, natural antioxidants have attracted attention for their safety, by preventing oxidative liver injuries, and the dietary consumption of antioxidants is recommended to prevent liver diseases [18]. Thus, we investigated the hepatoprotective effect of MES on $t$-BHP-treated HepG2 cells. The results showed that MES increased the viability of the $t$-BHP-treated cells by reducing the level of ROS and MDA and by increasing the level of GSH. Moreover, MES induced the expression of GST, HO-1, and NQO1 via the translocation of Nrf2 into the nucleus of the $t$-BHP-treated cells. The results also demonstrated that MES exhibits a hepatoprotective activity through its intrinsic antioxidant activity and by inducing detoxifying enzymes via the activation of Nrf2 in $t$-BHP-treated HepG2 cells.

T-BHP is a well-known oxidant that is used as a model compound to induce acute oxidative stress in vivo and in vitro [19]. It can be metabolized to free radical intermediates by cytochrome $\mathrm{P} 450$ in hepatocytes. The generated free radicals in the mitochondria are primarily eliminated by GSH, thus preventing damage to the lipids, proteins, and DNA [20]. Glutathione is a key antioxidant molecule against oxidative stress, and it can scavenge reactive radicals by conjugation and hydroperoxide reduction [21,22]. The reaction of ROS with the double bonds in polyunsaturated fatty acids produced lipid hydroperoxides, which form MDA by the degradation of peroxidized PUFAs. A high level of MDA caused by oxidative damages has been associated with various diseases, and it has been commonly been used as an index of lipid peroxidation [23]. The protective effect of MES on lipid peroxidation in HepG2 cells observed in the present study is consistent with the results of previous studies, which reported a similar effect by tea catechins [24], melanoidin [23], and quercetin [25] in the same cell line. These results showed that the MES-mediated protection against $t$-BHP-induced hepatotoxicity might be due to the intrinsic antioxidant activity of MES.

Oxidative damage is one of the primary factors involved in the development of hepatopathy, including hepatitis and cirrhosis. HepG2 cells maintain an antioxidant defense system against the harmful effects of ROS [8]. In addition to the cellular antioxidant GSH, antioxidant enzymes SOD and catalase play a crucial role in the prevention of oxidative damage [26,27]. Superoxide dismutase can specifically catalyze the dismutation of superoxide radical anion to hydrogen peroxide and oxygen [28]. The resulting hydrogen peroxide is transformed to water and molecular oxygen by catalase. Thus, the alteration in the activity of antioxidant enzymes, such as SOD and catalase, might help protect 
against oxidative stress. In line with this, the tocotrienol-rich fraction from grape seeds suppressed the production of ROS and MDA [29]. Moreover, the fraction decreased the activity of the SOD and catalase in the $t$-BHP-treated HepG2 cells [29]. The decrease in the activity of SOD and catalase by MES in the $t$-BHP-treated HepG2 cells observed in the present study was presumed to be due to the reduced ROS and enhanced GSH levels.

Several studies have shown that the hepatoprotective effect of natural compounds might be associated with the inhibition of oxidative stress by enhancing the antioxidant defense system and their intrinsic antioxidant activities [7,30]. Under normal conditions, Nrf2 sequestered by Keap1 is mostly distributed in the cytosol in an inactive form. Elevated levels of ROS by $t$-BHP treatment stimulates the Nrf2 activation, which regulates the cellular redox balance [7,31]. Activated Nrf2 binds to the antioxidant response element in the promoter regions of the antioxidants and detoxifying enzymes, including GST, HO-1, and NQO1 [32]. Glutathione S-transferase detoxifies the toxic substances by the enzymatic conjugation of reduced glutathione and xenobiotic substrates [26]. Heme oxygenase-1 catalyzes the degradation of heme to produce carbon monoxide and biliverdin, which is subsequently converted into bilirubin [7]. As a result, carbon monoxide and bilirubin act as strong antioxidants. NQO1 is an inducible protein under a variety of stress conditions, including oxidative stress, and plays multiple roles in cellular adaptation to oxidative stress [31]. Recent studies have reported that GST, $\mathrm{HO}-1$, and NQO1 are induced by various phytochemicals via the activation of Nrf2, to combat oxidative damage $[27,30]$. The results of the present study showed that Nrf2 is translocated into the nucleus, upregulating the downstream detoxifying enzymes including NQO1, HO-1, and GST, evidenced by fluorescence assay and western blotting. This suggests that MES protects oxidative damage by stimulating the production of antioxidants and by detoxifying enzymes via Nrf2 activation and its intrinsic antioxidant activity in the $t$-BHP-treated HepG2 cells.

In our previous paper, we found that MES prevented diet-induced obesity and hepatic steatosis [15]. The major components for inhibiting the triglyceride (TG) accumulation in adipocytes were identified as SHQA, SCM, and SQA, which showed two or three times lower $\mathrm{IC}_{50}$ values than MES. However, the inhibitory effect of MES on diet-induced TG accumulation in hepatocytes was mainly caused by SCM and SQA, but SHQA did not potently contribute to the inhibition of TG accumulation in hepatocytes. Thus, the inhibition of the lipid accumulation in adipocytes was principally caused by SHQA, SCM, and SQA, however, that in the hepatocytes was caused by SCM, SQA, and unidentified components in MES. Additionally, MES inhibited the expression of intracellular adhesion molecule 1 (ICAM-1) and vascular cell adhesion molecule 1 (VCAM-1) in high cholesterol died (HCD)-fed mice [16]. SHQA showed an 8.8-fold higher inhibitory activity on the expression of VCAM-1 than MES, however, it was not effective in the inhibition of the ICAM-1 expression in TNF- $\alpha$-treated HUVECs, suggesting selective effects of VCAM-1 [16]. This study investigates the antioxidant activity of MES and evaluates its hepatoprotective effect on $t$-BHP-treated HepG2 cells, as MES contains high levels of SHQA, SCMs and SQA. In this study, the $\mathrm{IC}_{50}$ value of MES and SCM for scavenging ROS radicals was similar, whereas, that of SHQA and SQA were less than MES. Based on the $\mathrm{IC}_{50}$ values and the content of the three compounds, SHQA is the main antioxidant component in MES. Also, MES may contain a marginal antioxidant activity, which would be caused by unidentified compounds. Although SHQA and SQA showed high antioxidant activity compared with MES, MES has an advantage for the commercial utilization of an antioxidant agent, because the isolation of each component requires a high cost and complicated procedure. This is an initiating study to assess the potency of S. serratifolium for hepatoprotection. MES showed cytoprotective properties by regulating the biochemical/molecular markers related to oxidative stress in the $t$-BHP treated cells, important pharmacological properties are largely uncentain. Further animal studies are required to identify the molecular target(s) of MES and its active components. 


\section{Materials and Methods}

\subsection{Materials}

HepG2 cell line and Eagle's minimum essential medium (EMEM) were purchased from ATCC (Manassas, VA, USA). Fetal bovine serum (FBS) and 0.25\% trypsin-EDTA were purchased from Gibco-BRL Life Technologies (Grand Island, NJ, USA). The CellTiter96 Aqueous One Solution Cell Proliferation Assay kit was obtained from Promega (Madison, WI, USA). 5-Chloromethylfluorescein diacetate (CMF-DA), 4'6-diamidino-2-phenylindole (DAPI), protein marker, enhanced chemiluminescence (ECL), NE-PER Nuclear and Cytoplasmic Extraction Reagents, and the BCA protein assay kit were obtained from Thermo (Waltham, MA, USA). Tert-butyl hydroperoxide (t-BHP), 2,7-dichlorofluorescein diacetate (DCFH-DA), dimethyl sulfoxide (DMSO), and N-acetyl-cysteine (NAC) were purchased from Sigma-Aldrich (St. Louis, MO, USA). The antibodies against HO-1 (ab13248), NQO1 (ab34173), and anti-Nrf2 antibody (ab206893) were obtained from Abcam (Cambridge, UK). $\beta$-Actin (sc-47778), PARP (sc-7150), Nrf2 (sc-365949), and horseradish peroxidase-conjugated secondary antibodies (sc-2031) were purchased from Santa Cruz Biotechnology (Santa Cruz, CA, USA). The superoxide dismutase (SOD), catalase, and glutathione S-transferase (GST) assay kit were purchased from Cayman Chemical Co. (Ann Arbor, MI, USA).

\subsection{Preparation of MES and Isolation of Chemical Components}

S. serratifolium was collected along the coast of Busan, South Korea, in May 2017. The specimen identity was confirmed by an algal taxonomist (C.G. Choi) at the Department of Ecological Engineering, Pukyong National University, Republic of Korea. The collected sample was air-dried and ground. One and half kilograms of the dried sample were extracted twice, with $70 \%$ ethanol (6 L each time) at $70{ }^{\circ} \mathrm{C}$ for $3 \mathrm{~h}$. The combined extract was filtered with an ultrafiltration unit (MWCO, $\left.50 \mathrm{kDa}\right)$ and was concentrated until a lipophilic fraction was separated from the salt water. The lipophilic fraction was concentrated by a rotary vacuum evaporator (Eyela N3010, Tokyo, Japan) at $45{ }^{\circ} \mathrm{C}$ until the water content was less than 5.5\%, and was used for this study. From the $1.5 \mathrm{~kg}$ of dried sample, $120 \mathrm{~g}$ of the MES was obtained. The isolation and quantification of SHQA, SCM, and SQA were performed according to the method described previously [14,33].

\subsection{Cell Culture and Viability Assay}

The HepG2 cells (ATCC, Manassas, VA, USA) were cultured in EMEM media containing 10\% FBS in a humidified atmosphere of $5 \% \mathrm{CO}_{2}$. The HepG2 cells were plated in a 96-well microplate $\left(4 \times 10^{4}\right.$ cells/well) and incubated for $24 \mathrm{~h}$. The culture media were replaced by $100 \mu \mathrm{L}$ of MES $(2.5,5.0$ and $10.0 \mu \mathrm{g} / \mathrm{mL})$, diluted with a culture medium, and then incubated for $24 \mathrm{~h}$. The cell viability was measured by CellTiter96 Aqueous One Solution Cell Proliferation Assay kit, according to manufacturer's instructions. After $1 \mathrm{~h}$ of incubation at $37^{\circ} \mathrm{C}$, the plate was measured with a microplate reader (GloMax-multi detection system, Promega, Madison, WI, USA) at $490 \mathrm{~nm}$. The MES stock solution $(100 \mathrm{ug} / \mathrm{mL})$ was made by dissolving in DMSO, and the working solution was made with a culture medium by diluting with a culture medium to obtain appropriate concentration.

\subsection{Determination of ROS Production}

Intracellular ROS level was determined by the oxidant-sensitive fluorescent probe DCFH-DA, as described previously [27]. The HepG2 cells were plated in a 96-well microplate $\left(4 \times 10^{4}\right.$ cells $/$ well $)$, and then incubated for $24 \mathrm{~h}$. The cells were treated with MES $(0.25,0.5$, and $1.0 \mu \mathrm{g} / \mathrm{mL})$ diluted with a culture medium, or MES and $0.5 \mathrm{mM}$ of $t$-BHP, and then incubated for $1 \mathrm{~h}$. The media was changed with $20 \mu \mathrm{M}$ of DCFH-DA solution and incubated at $37{ }^{\circ} \mathrm{C}$ for $30 \mathrm{~min}$. The fluorescence intensity was measured at an excitation wavelength of $485 \mathrm{~nm}$ and the emission wavelength of $523 \mathrm{~nm}$, using amicroplate reader. 


\subsection{Measurement of Lipid Peroxidation}

The lipid peroxidation was determined with a malondialdehyde (MDA) concentration using a thiobarbituric acid reactive substance (TBARS) assay kit from Cayman Chemical Company (Ann Arbor, MI, USA). Briely, the HepG2 cells were plated in six-well plates $\left(1.2 \times 10^{6}\right.$ cells/well), and were then treated with an indicated concentration of MES and $0.5 \mathrm{mM} t$-BHP for $24 \mathrm{~h}$ to give enough exposing time for the MES. After treatment, the cells were scraped off and suspended in PBS. The suspended cells were sonicated and measured the concentration of MDA according to the manufacturer's instructions. The absorbance of the product was measured at $540 \mathrm{~nm}$ using a microplate reader. The result was expressed as micromoles of MDA equivalents TBARS per microgram of protein.

\subsection{Determination of Glutathione Level}

The intracellular glutathione concentration was determined using the fluorescent probe CMF-DA, described in our previous research [27]. The HepG2 cells were plated in 96-well microplates $\left(5 \times 10^{4}\right.$ cells/well), and then incubated for $24 \mathrm{~h}$. After incubation, the media were changed with MES $(0.25,0.5$, and $1.0 \mu \mathrm{g} / \mathrm{mL})$, diluted with a culture medium, and $0.5 \mathrm{mM}$ of $t$-BHP for $1 \mathrm{~h}$. The media was changed with $10 \mu \mathrm{M}$ of CMF-DA solution, and incubated at $37^{\circ} \mathrm{C}$ for $1 \mathrm{~h}$. The fluorescence intensity was measured at an excitation wavelength of $485 \mathrm{~nm}$ and the emission wavelength of $523 \mathrm{~nm}$, using microplate reader.

\subsection{Measurement of Antioxidant Enzyme Activities}

The HepG2 cells cultured in six-well plates $\left(1.2 \times 10^{6}\right.$ cells/well $)$ were treated with the indicated concentration of MES and $0.5 \mathrm{mM}$ of $t$-BHP for $18 \mathrm{~h}$, since the change of the antioxidant enzyme activities were detectable after $18 \mathrm{~h}$ of MES treatment. After incubation, the cells were washed and harvested with a rubber scraper. The cells were suspended in PBS and sonicated for $10 \mathrm{~s}$ on ice. The cell lysates were centrifuged at $10,000 \times g$ for $15 \mathrm{~min}$. The supernatants were stored at $-80{ }^{\circ} \mathrm{C}$, until required by the experiments. $\mathrm{N}$-acetyl-cysteine (NAC) was used as a positive control. The catalase, SOD, and GST activities were measured using the Cayman Assay kit, according to the manufacturer's instructions.

\subsection{Separation of Nuclear and Cytosolic Extract}

The HepG2 cells $\left(1.2 \times 10^{6}\right.$ cell/well $)$ were treated with indicated concentrations MES and $0.5 \mathrm{mM}$ of $t$-BHP for $1 \mathrm{~h}$. The cells were washed with ice-cold PBS. The harvested cells were centrifuged at $1000 \times g$ for $5 \mathrm{~min}$ at $4{ }^{\circ} \mathrm{C}$. The nuclear and cytosolic fractions were separated using NE-PER Nuclear and Cytoplasmic Extraction Reagent (Thermo, Waltham, MA, USA), according to our previous paper [30]. The separated fractions were stored at $-70{ }^{\circ} \mathrm{C}$, until further use.

\subsection{Western Blotting}

The HepG2 cells cultured in six-well culture plates $\left(1.2 \times 10^{6}\right.$ cells / well $)$ were treated with the indicated concentrations of MES and $0.5 \mathrm{mM}$ of $t$-BHP for $18 \mathrm{~h}$. The cells were lysed with a lysis buffer (50 mM Tris- $\mathrm{HCl}$, pH 7.5, $150 \mathrm{mM} \mathrm{NaCl}, 1 \% \mathrm{NP}-40,1 \%$ Tween-20, 0.1\% SDS, 1 mM Na $3 \mathrm{VO}_{4}, 10 \mu \mathrm{g} / \mathrm{mL}$ leupeptin, $50 \mathrm{mM} \mathrm{NaF}$, and $1 \mathrm{mM} \mathrm{PMSF}$ ) on ice for $30 \mathrm{~min}$. After incubation, the cell lysates were centrifuged at $12,000 \times g$ for $20 \mathrm{~min}$, the supernatants were transferred and determined the protein concentration using a BCA protein assay kit (Thermo, Waltham, MA, USA). Aliquots of proteins $(40 \mu \mathrm{g})$ were separated by SDS-PAGE and were transferred onto a nitrocellulose membrane (Millipore, Burlington, MA, USA). The membrane was incubated with a primary antibody for $2 \mathrm{~h}$. After washing with TBST, the membrane was treated with horseradish peroxidase-conjugated secondary antibody for $1 \mathrm{~h}$. The proteins were detected using an ECL detection reagent. A densitometric analysis of the data was performed using a cooled CCD camera system EZ-Capture II and CS analyzer version 3.00 software (ATTO Co., Tokyo, Japan). 


\subsection{Immunofluorescence Analysis}

To determine the nuclear localization of Nrf2 in the HepG2 cells, the cells were cultured on eight-well chamber slides (SPL Life Sciences Co., Gyeonggi-do, Korea) for $24 \mathrm{~h}$, and were treated with $0.5 \mu \mathrm{g} / \mathrm{mL}$ of MES and $0.5 \mathrm{mM}$ of $t$-BHP for $1 \mathrm{~h}$ at $37^{\circ} \mathrm{C}$. The treated cells were fixed in $4.0 \%(w / v)$ paraformaldehyde for $15 \mathrm{~min}$ at room temperature, and then permeabilized with $0.5 \%(v / v)$ Triton X-100 in PBS for 10 min. The permeabilized cells were blocked with $3 \%$ BSA in PBS for $1 \mathrm{~h}$. The cells were incubated overnight in an anti-Nrf2 antibody (Alexa Fluor 568, Abcam, Cambridge, UK) at $4{ }^{\circ} \mathrm{C}$. Then, the nuclei were stained with $2 \mu \mathrm{g} / \mathrm{mL}$ DAPI and observed using a LSM700 Laser scanning confocal microscope (Carl Zeiss, Oberkochen, Germany).

\subsection{Statistical Analysis}

All of the data were analyzed as the mean \pm standard deviation (SD) of three independent experiments, unless otherwise indicated. The data analysis was performed using ANOVA, followed by the Bonferroni test. $p<0.05$ were considered as statistically significant. SPSS for Windows, version 10.07 (SPAA Inc., Chicago, IL, USA), was used for the analyses.

\section{Conclusions}

We have demonstrated that MES suppresses the production of ROS and MDA, and increases the cellular level of GSH in $t$-BHP-treated HepG2 cells. It also induces the production of GST, HO-1, and NQO1. Although MES showed preferential properties by regulating the antioxidant markers related to oxidative stress, important pharmacological profiles are largely unknown. Specifically, it is important to identify the molecular target(s) of MES and its active components. The active compounds have different ROS scavenging activities in the $t$-BHP-treated cells; however, the individual targets for antioxidation are uncertain. Hence, it will be critical to further investigate molecular and biochemical targets in order to better understand the pharmacological and toxicological profiles of the active components

Author Contributions: S.L. and M.K. conducted most of experiments; E-J.J. and T.S. prepared MES and isolated SHQA, SCM, and SQA from MES; C.-W.O. and J.S.C. contributed to data collection and analysis; H.-R.K. supervised the entire project and had a major role in the experimental design, data interpretation, and writing the manuscript. All authors read and approved the final manuscript.

Funding: This study was a part of the projects, "Development of functional food products with natural materials derived from marine resources" [20170285] and "Development of nutraceuticals from Sargassum serratifolium" [20150311], funded by the Ministry of Oceans and Fisheries, Republic of Korea.

Acknowledgments: Eun-Ji Joung is supported by National Research Foundation of Korea (201 8R1D1A1B07049801). The authors are grateful to Chang-Geun Choi for his taxonomic identification of Sargassu serratifolium.

Conflicts of Interest: The authors declare no conflict of interest.

\section{References}

1. Reuter, S.; Gupta, S.C.; Chaturvedi, M.M.; Aggarwal, B.B. Oxidative stress, inflammation, and cancer: How are they linked? Free. Radic. Biol. Med. 2010, 49, 1603-1616. [CrossRef] [PubMed]

2. Kim, Y.; Choi, Y.; Ham, H.; Jeong, H.S.; Lee, J. Protective effects of oligomeric and polymeric procyanidin fractions from defatted grape seeds on tert-butyl hydroperoxide-induced oxidative damage in HepG2 cells. Food Chem. 2013, 137, 136-141. [CrossRef] [PubMed]

3. Chakraborty, K.; Joseph, D.; Praveen, N.K. Antioxidant activities and phenolic contents of three red seaweeds (Division: Rhodophyta) harvested from the Gulf of Mannar of Peninsular India. J. Food Sci. Technol. 2015, 52, 1924-1935. [CrossRef] [PubMed]

4. Kumar, H.; Kim, I.S.; More, S.V.; Kim, B.W.; Choi, D.K. Natural product-derived pharmacological modulators of Nrf2/ARE pathway for chronic diseases. Nat. Prod. Rep. 2014, 31, 109-139. [CrossRef] [PubMed]

5. Bryan, H.K.; Olayanju, A.; Goldring, C.E.; Park, B.K. The Nrf2 cell defence pathway: Keap1-dependent and -independent mechanisms of regulation. Biochem. Pharmacol. 2013, 85, 705-717. [CrossRef] [PubMed] 
6. Ross, D.; Siegel, D. NAD(P)H: Quinone Oxidoreductase 1 (NQO1, DT-Diaphorase), Functions and Pharmacogenetics. Methods Enzymol. 2004, 382, 115-144. [PubMed]

7. Loboda, A.; Damulewicz, M.; Pyza, E.; Jozkowicz, A.; Dulak, J. Role of Nrf2/HO-1 system in development, oxidative stress response and diseases: An evolutionarily conserved mechanism. Cell. Mol. Life Sci. 2016, 73, 3221-3247. [CrossRef] [PubMed]

8. Choi, J.S.; Han, Y.R.; Byeon, J.S.; Choung, S.Y.; Sohn, H.S.; Jung, H.A. Protective effect of fucosterol isolated from the edible brown algae, Ecklonia stolonifera and Eisenia bicyclis, on tert-butyl hydroperoxide- and tacrine-induced HepG2 cell injury. J. Pharm. Pharmacol. 2015, 67, 1170-1178. [CrossRef] [PubMed]

9. Kucera, O.; Endlicher, R.; Rousar, T.; Lotkova, H.; Garnol, T.; Drahota, Z.; Cervinkova, Z. The effect of tert-butyl hydroperoxide-induced oxidative stress on lean and steatotic rat hepatocytes in vitro. Oxid. Med. Cell. Longev. 2014, 2014, 752506. [CrossRef] [PubMed]

10. Knasmüller, S.; Parzefall, W.; Sanyal, R.; Ecker, S.; Schwab, C.; Uhl, M.; Mersch-Sundermann, V.; Williamson, G.; Hietsch, G.; Langer, T.; et al. Use of metabolically competent human hepatoma cells for the detection of mutagens and antimutagens. Mutat. Res.-Fundam. Mol. Mech. Mutagen. 1998, 402, 185-202. [CrossRef]

11. Liu, L.; Heinrich, M.; Myers, S.; Dworjanyn, S.A. Towards a better understanding of medicinal uses of the brown seaweed Sargassum in Traditional Chinese Medicine: A phytochemical and pharmacological review. J. Ethnopharmacol. 2012, 142, 591-619. [CrossRef] [PubMed]

12. Balboa, E.M.; Conde, E.; Moure, A.; Falque, E.; Dominguez, H. In vitro antioxidant properties of crude extracts and compounds from brown algae. Food Chem. 2013, 138, 1764-1785. [CrossRef] [PubMed]

13. Oh, S.J.; Joung, E.J.; Kwon, M.S.; Lee, B.; Utsuki, T.; Oh, C.W.; Kim, H.R. Anti-inflammatory effect of ethanolic extract of Sargassum serratifolium in lipopolysaccharide-stimulated BV2 microglial cells. J. Med. Food 2016, 19, 1023-1031. [CrossRef] [PubMed]

14. Joung, E.J.; Gwon, W.G.; Shin, T.; Jung, B.M.; Choi, J.; Kim, H.R. Anti-inflammatory action of the ethanolic extract from Sargassum serratifolium on lipopolysaccharide-stimulated mouse peritoneal macrophages and identification of active components. J. Appl. Phycol. 2017, 29, 563-573. [CrossRef]

15. Kwon, M.; Lim, S.J.; Joung, E.J.; Lee, B.; Oh, C.W.; Kim, H.R. Meroterpenoid-rich fraction of an ethanolic extract from Sargassum serratifolium alleviates obesity and non-alcoholic fatty liver disease in high fat-fed C57BL/6J mice. J. Funct. Foods 2018, 47, 288-298. [CrossRef]

16. Gwon, W.G.; Joung, E.J.; Shin, T.; Utsuki, T.; Wakamatsu, N.; Kim, H.-R. Meroterpinoid-rich fraction of the ethanol extract from Sargassum serratifolium suppresses TNF- $\alpha$-induced monocytes adhesion to vascular endothelium and vascular inflammation in high cholesterol-fed C57BL/6J mice. J. Funct. Foods 2018, 46, 384-393. [CrossRef]

17. Zhu, R.Z.; Wang, Y.J.; Zhang, L.Q.; Guo, Q.L. Oxidative stress and liver disease. Hepatol. Res. 2012, 42, 741-749. [CrossRef] [PubMed]

18. Huang, Z.Q.; Chen, P.; Su, W.W.; Wang, Y.G.; Wu, H.; Peng, W.; Li, P.B. Antioxidant Activity and Hepatoprotective Potential of Quercetin 7-Rhamnoside In Vitro and In Vivo. Molecules 2018, 23, 1188. [CrossRef] [PubMed]

19. Yang, S.Y.; Hong, C.O.; Lee, G.P.; Kim, C.T.; Lee, K.W. The hepatoprotection of caffeic acid and rosmarinic acid, major compounds of Perilla frutescens, against t-BHP-induced oxidative liver damage. Food Chem. Toxicol. 2013, 55, 92-99. [CrossRef] [PubMed]

20. Noh, J.R.; Gang, G.T.; Kim, Y.H.; Yang, K.J.; Hwang, J.H.; Lee, H.S.; Oh, W.K.; Song, K.S.; Lee, C.H. Antioxidant effects of the chestnut (Castanea crenata) inner shell extract in t-BHP-treated HepG2 cells, and CCl4- and high-fat diet-treated mice. Food Chem. Toxicol. 2010, 48, 3177-3183. [CrossRef] [PubMed]

21. Wu, G.; Fang, Y.Z.; Yang, S.; Lupton, J.R.; Turner, N.D. Glutathione Metabolism and Its Implications for Health. J. Nutr. 2004, 134, 489-492. [CrossRef] [PubMed]

22. Hwang, Y.P.; Choi, J.H.; Choi, J.M.; Chung, Y.C.; Jeong, H.G. Protective mechanisms of anthocyanins from purple sweet potato against tert-butyl hydroperoxide-induced hepatotoxicity. Food Chem. Toxicol. 2011, 49, 2081-2089. [CrossRef] [PubMed]

23. Goya, L.; Delgado-Andrade, C.; Rufian-Henares, J.A.; Bravo, L.; Morales, F.J. Effect of coffee melanoidin on human hepatoma HepG2 cells. Protection against oxidative stress induced by tert-butylhydroperoxide. Mol. Nutr. Food Res. 2007, 51, 536-545. [CrossRef] [PubMed] 
24. Chen, L.; Yang, X.; Jiao, H.; Zhao, B. Tea catechins protect against lead-induced cytotoxicity, lipid peroxidation, and membrane fluidity in HepG2 cells. Toxicol. Sci. 2002, 69, 149-156. [CrossRef] [PubMed]

25. Alia, M.; Ramos, S.; Mateos, R.; Granado-Serrano, A.B.; Bravo, L.; Goya, L. Quercetin protects human hepatoma HepG2 against oxidative stress induced by tert-butyl hydroperoxide. Toxicol. Appl. Pharmacol. 2006, 212, 110-118. [CrossRef] [PubMed]

26. Atkinson, H.J.; Babbitt, P.C. Glutathione transferases are structural and functional outliers in the thioredoxin fold. Biochemistry 2009, 48, 11108-11116. [CrossRef] [PubMed]

27. Lee, M.S.; Lee, B.; Park, K.E.; Utsuki, T.; Shin, T.; Oh, C.W.; Kim, H.R. Dieckol enhances the expression of antioxidant and detoxifying enzymes by the activation of Nrf2-MAPK signalling pathway in HepG2 cells. Food Chem. 2015, 174, 538-546. [CrossRef] [PubMed]

28. Waddington, R.J.; Moseley, R.; Embery, G. Reactive oxygen species: A potential role in the pathogenesis of periodontal diseases. Oral Dis. 2000, 6, 138-151. [CrossRef] [PubMed]

29. Choi, Y.; Lee, S.-M.; Kim, Y.; Yoon, J.; Jeong, H.-S.; Lee, J. A tocotrienol-rich fraction from grape seeds inhibits oxidative stress induced by tert-butyl hydroperoxide in HepG2 cells. J. Med. Food 2010, 13, 1240-1246. [CrossRef] [PubMed]

30. Jun, Y.J.; Lee, M.; Shin, T.; Yoon, N.; Kim, J.H.; Kim, H.R. Eckol enhances heme oxygenase-1 expression through activation of Nrf2/JNK pathway in HepG2 cells. Molecules 2014, 19, 15638-15652. [CrossRef] [PubMed]

31. Ross, D.; Siegel, D. Functions of NQO1 in cellular protection and CoQ10 metabolism and its potential role as a redox sensitive molecular switch. Front. Physiol. 2017, 8, 595. [CrossRef] [PubMed]

32. Huo, X.; Liu, C.; Gao, L.; Xu, X.; Zhu, N.; Cao, L. Hepatoprotective effect of aqueous extract from the seeds of orychophragmus violaceus against liver injury in mice and HepG2 cells. Int. J. Mol. Sci. 2017, 18, 1197. [CrossRef] [PubMed]

33. Azam, M.S.; Joung, E.J.; Choi, J.; Kim, H.R. Ethanolic extract from Sargassum serratifolium attenuates hyperpigmentation through CREB/ERK signaling pathways in $\alpha$-MSH-stimulated B16F10 melanoma cells. J. Appl. Phycol. 2017, 29, 2089-2096. [CrossRef]

(C) 2018 by the authors. Licensee MDPI, Basel, Switzerland. This article is an open access article distributed under the terms and conditions of the Creative Commons Attribution (CC BY) license (http:/ / creativecommons.org/licenses/by/4.0/). 